\title{
Meridian Surfaces on Rotational Hypersurfaces with Lightlike Axis in $\mathbb{E}_{2}^{4}$
}

\author{
Velichka Milousheva
}

Velichka Milousheva: Institute of Mathematics and Informatics, Bulgarian Academy of Sciences, Acad. G. Bonchev Str. bl. 8, 1113, Sofia, Bulgaria, e-mail:vmil@math.bas.bg

\begin{abstract}
We construct a special class of Lorentz surfaces in the pseudoEuclidean 4-space with neutral metric which are one-parameter systems of meridians of rotational hypersurfaces with lightlike axis and call them meridian surfaces. We give the complete classification of the meridian surfaces with constant Gauss curvature and prove that there are no meridian surfaces with parallel mean curvature vector field other than CMC surfaces lying in a hyperplane. We also classify the meridian surfaces with parallel normalized mean curvature vector field. We show that in the family of the meridian surfaces there exist Lorentz surfaces which have parallel normalized mean curvature vector field but not parallel mean curvature vector.
\end{abstract}

Keywords. Meridian surfaces - constant Gauss curvature · parallel mean curvature vector - parallel normalized mean curvature vector.

MSC 2010 Classification. Primary: 53A35; Secondary:53B30 - 53B25.

\section{$1 \quad$ INTRODUCTION}

A fundamental problem of the contemporary differential geometry of surfaces and hypersurfaces in standard model spaces such as the Euclidean space $\mathbb{E}^{n}$ and the pseudo-Euclidean space $\mathbb{E}_{k}^{n}$ is the investigation of the basic invariants characterizing the surfaces. Curvature invariants are the number one Riemannian invariants and the most natural ones. The basic intrinsic curvature invariant of a surface in 4-dimensional Euclidean or pseudo-Euclidean space is the Gauss curvature and one basic extrinsic invariant is the curvature of the normal connection. The most important normal vector field of a surface is the mean curvature vector field. So, a fundamental question is to investigate various important classes of surfaces characterized by conditions on the Gauss curvature, the normal curvature, or the mean curvature vector field, and to find examples of surfaces belonging to these classes.

Rotational surfaces and hypersurfaces are basic source of examples of many geometric classes of surfaces in Riemannian and pseudo-Riemannian geometry. The main purpose of this paper is to provide a comprehensive survey on a 
special class of surfaces (called meridian surfaces) in 4-dimensional Euclidean or pseudo-Euclidean spaces which are one-parameter systems of meridians of rotational hypersurfaces. We present briefly recent results on meridian surfaces in the Euclidean space $\mathbb{E}^{4}$ and the Minkowski space $\mathbb{E}_{1}^{4}$.

In the present paper, the new contribution to the theory of meridian surfaces is the construction of 2-dimensional Lorentz surfaces in the pseudo-Euclidean space $\mathbb{E}_{2}^{4}$ which are one-parameter systems of meridians of a rotational hypersurface with lightlike axis. They are analogous to the meridian surfaces lying on rotational hypersurfaces with spacelike or timelike axis in $\mathbb{E}_{2}^{4}$ which have been studied in [3] and [4]. We show that all meridian surfaces are surfaces with flat normal connection and classify completely the meridian surfaces with constant Gauss curvature (Theorem 4.1 and Theorem 4.2). In Theorem 5.1 we give the classification of the meridian surfaces with parallel mean curvature vector field $H$. Theorem 6.1 describes all meridian surfaces which have parallel normalized mean curvature vector field but not parallel $H$.

\section{PRELIMINARIES}

Let $\mathbb{E}_{2}^{4}$ be the 4-dimensional pseudo-Euclidean space with the canonical pseudoEuclidean metric of index 2 given in local coordinates by

$$
\widetilde{g}=d x_{1}^{2}+d x_{2}^{2}-d x_{3}^{2}-d x_{4}^{2}
$$

where $\left(x_{1}, x_{2}, x_{3}, x_{4}\right)$ is a rectangular coordinate system of $\mathbb{E}_{2}^{4}$. Denote by $\langle.,$. the indefinite inner scalar product associated with $\widetilde{g}$. Since $\widetilde{g}$ is an indefinite metric, a vector $v \in \mathbb{E}_{2}^{4}$ can have one of the three casual characters: spacelike if $\langle v, v\rangle>0$ or $v=0$, timelike if $\langle v, v\rangle<0$, and lightlike if $\langle v, v\rangle=0$ and $v \neq 0$. This terminology is inspired by general relativity and the Minkowski 4-space $\mathbb{E}_{1}^{4}$.

We use the following standard denotations:

$$
\begin{aligned}
& \mathbb{S}_{2}^{3}(1)=\left\{V \in \mathbb{E}_{2}^{4}:\langle V, V\rangle=1\right\} \\
& \mathbb{H}_{1}^{3}(-1)=\left\{V \in \mathbb{E}_{2}^{4}:\langle V, V\rangle=-1\right\} .
\end{aligned}
$$

The space $\mathbb{S}_{2}^{3}(1)$ is known as the de Sitter space, and the space $\mathbb{H}_{1}^{3}(-1)$ is the anti-de Sitter space [22].

A surface $M$ in $\mathbb{E}_{2}^{4}$ is called Lorentz, if $\langle.,$.$\rangle induces a Lorentzian metric g$ on $M$, i.e. at each point $p \in M$ we have the following decomposition

$$
\mathbb{E}_{2}^{4}=T_{p} M \oplus N_{p} M
$$

with the property that the restriction of the metric onto the tangent space $T_{p} M$ is of signature $(1,1)$, and the restriction of the metric onto the normal space $N_{p} M$ is of signature $(1,1)$. 
Denote by $\nabla$ and $\bar{\nabla}$ the Levi-Civita connections of $M$ and $\mathbb{E}_{2}^{4}$, respectively. For any tangent vector fields $X, Y$ and any normal vector field $\xi$, the Gauss formula and the Weingarten formula are given by

$$
\begin{aligned}
& \bar{\nabla}_{X} Y=\nabla_{X} Y+h(X, Y), \\
& \bar{\nabla}_{X} \xi=-A_{\xi} X+D_{X} \xi,
\end{aligned}
$$

where $h$ is the second fundamental form of $M, D$ is the normal connection on the normal bundle, and $A_{\xi}$ is the shape operator with respect to $\xi$.

The mean curvature vector field $H$ of $M$ in $\mathbb{E}_{2}^{4}$ is defined as $H=\frac{1}{2} \operatorname{tr} h$. A surface $M$ is called minimal if its mean curvature vector vanishes identically, i.e. $H=0$. A natural extension of minimal surfaces are quasi-minimal surfaces. A surface $M$ is called quasi-minimal (or pseudo-minimal) if its mean curvature vector is lightlike at each point, i.e. $H \neq 0$ and $\langle H, H\rangle=0$. In the Minkowski space $\mathbb{E}_{1}^{4}$ the quasi-minimal surfaces are also called marginally trapped. This notion is borrowed from general relativity. A surface $M$ is said to have constant mean curvature if $\langle H, H\rangle=$ const. We shall consider Lorentz surfaces in $\mathbb{E}_{2}^{4}$ for which $\langle H, H\rangle=$ const $\neq 0$. Such surfaces we call $C M C$ surfaces.

A normal vector field $\xi$ on $M$ is called parallel in the normal bundle (or simply parallel) if $D \xi=0$ holds identically [7]. A surface $M$ is said to have parallel mean curvature vector field if its mean curvature vector $H$ satisfies $D H=0$.

Surfaces for which the mean curvature vector field $H$ is non-zero, $\langle H, H\rangle \neq 0$, and there exists a unit vector field $H_{0}$ in the direction of the mean curvature vector $H$, such that $H_{0}$ is parallel in the normal bundle, are called surfaces with parallel normalized mean curvature vector field [6]. It is easy to see that if $M$ is a surface with non-zero parallel mean curvature vector field $H$ (i.e. $D H=0$ ), then $M$ is a surface with parallel normalized mean curvature vector field, but the converse is not true in general. It is true only for surfaces with $\|H\|=$ const.

\section{Construction of Meridian Surfaces in Pseudo-Euclidean 4-Space}

Meridian surfaces in the Euclidean 4-space $\mathbb{E}^{4}$ we defined in [15] as one-parameter systems of meridians of the standard rotational hypersurface in $\mathbb{E}^{4}$. The classification of meridian surfaces with constant Gauss curvature, with constant mean curvature, Chen meridian surfaces and meridian surfaces with parallel normal bundle is given in [15] and [17]. The meridian surfaces in $\mathbb{E}^{4}$ with pointwise 1-type Gauss map are classified in [1]. The idea from the Euclidean space is used in [16], [18], and [19] for the construction of meridian spacelike surfaces lying on rotational hypersurfaces in $\mathbb{E}_{1}^{4}$ with timelike, spacelike, or lightlike axis. The classification of marginally trapped meridian surfaces is given in [16] and [19]. Meridian surfaces in $\mathbb{E}_{1}^{4}$ with pointwise 1-type Gauss map are classified in 
[2]. The classification of meridian surfaces with constant Gauss curvature, with constant mean curvature, Chen meridian surfaces and meridian surfaces with parallel normal bundle is given in [18] and [20].

Following the idea from the Euclidean and Minkowski spaces, in [3] and [4] we constructed Lorentz meridian surfaces in the pseudo-Euclidean 4 -space $\mathbb{E}_{2}^{4}$ as one-parameter systems of meridians of rotational hypersurfaces with timelike or spacelike axis. We gave the classification of quasi-minimal meridian surfaces and meridian surfaces with constant mean curvature [3]. The classification of meridian surfaces with parallel mean curvature vector field and the classification of meridian surfaces with parallel normalized mean curvature vector is given in $[4]$.

In the present paper we construct Lorentz meridian surfaces in $\mathbb{E}_{2}^{4}$ which are one-parameter systems of meridians of rotational hypersurfaces with lightlike axis.

Let $O e_{1} e_{2} e_{3} e_{4}$ be a fixed orthonormal coordinate system in $\mathbb{E}_{2}^{4}$, i.e. $\left\langle e_{1}, e_{1}\right\rangle=$ $\left\langle e_{2}, e_{2}\right\rangle=1,\left\langle e_{3}, e_{3}\right\rangle=\left\langle e_{4}, e_{4}\right\rangle=-1$. We denote $\xi_{1}=\frac{e_{2}+e_{4}}{\sqrt{2}}, \xi_{2}=\frac{-e_{2}+e_{4}}{\sqrt{2}}$ and consider the pseudo-orthonormal base $\left\{e_{1}, e_{3}, \xi_{1}, \xi_{2}\right\}$ of $\mathbb{E}_{2}^{4}$. Note that $\left\langle\xi_{1}, \xi_{1}\right\rangle=0,\left\langle\xi_{2}, \xi_{2}\right\rangle=0,\left\langle\xi_{1}, \xi_{2}\right\rangle=-1$.

A rotational hypersurface with lightlike axis in $\mathbb{E}_{2}^{4}$ can be parametrized by

$\mathcal{M}: Z\left(u, w^{1}, w^{2}\right)=f(u) w^{1}\left(\cosh w^{2} e_{1}+\sinh w^{2} e_{3}\right)+\left(f(u) \frac{\left(w^{1}\right)^{2}}{2}+g(u)\right) \xi_{1}+f(u) \xi_{2}$,

where $f=f(u), g=g(u)$ are smooth functions, defined in an interval $I \subset \mathbb{R}$ and $f(u)>0, u \in I$.

Let $w^{1}=w^{1}(v), w^{2}=w^{2}(v), v \in J, J \subset \mathbb{R}$ and consider the surface $\mathcal{M}_{m}$ in $\mathbb{E}_{2}^{4}$ given by

$$
\mathcal{M}_{m}: z(u, v)=Z\left(u, w^{1}(v), w^{2}(v)\right),
$$

where $u \in I, v \in J$. The surface $\mathcal{M}_{m}$, defined by (3.1), is a one-parameter system of meridians of the rotational hypersurface $\mathcal{M}$. So, we call $\mathcal{M}_{m}$ a meridian surface on $\mathcal{M}$.

Without loss of generality we can assume that $w^{1}=\varphi(v), w^{2}=v$. Then the meridian surface $\mathcal{M}_{m}$ is parametrized as follows:

$$
\mathcal{M}_{m}: z(u, v)=f(u)\left(\varphi(v) \cosh v e_{1}+\varphi(v) \sinh v e_{3}+\frac{\varphi^{2}(v)}{2} \xi_{1}+\xi_{2}\right)+g(u) \xi_{1} .
$$

If we denote $l(v)=\varphi(v) \cosh v e_{1}+\varphi(v) \sinh v e_{3}+\frac{\varphi^{2}(v)}{2} \xi_{1}+\xi_{2}$, then the parametrization (3.2) is written as

$$
\mathcal{M}_{m}: z(u, v)=f(u) l(v)+g(u) \xi_{1} .
$$

Now we shall find the coefficients of the first fundamental form of $\mathcal{M}_{m}$. The 
tangent vector fields $z_{u}$ and $z_{v}$ are

$$
\begin{aligned}
& z_{u}=f^{\prime} \varphi \cosh v e_{1}+f^{\prime} \varphi \sinh v e_{3}+\left(f^{\prime} \frac{\varphi^{2}}{2}+g^{\prime}\right) \xi_{1}+f^{\prime} \xi_{2} \\
& z_{v}=f(\dot{\varphi} \cosh v+\varphi \sinh v) e_{1}+f(\dot{\varphi} \sinh v+\varphi \cosh v) e_{3}+f \varphi \dot{\varphi} \xi_{1},
\end{aligned}
$$

where $\dot{\varphi}$ denotes the derivative of $\varphi$ with respect to $v$. So, the coefficients of the first fundamental form are

$$
E=-2 f^{\prime}(u) g^{\prime}(u) ; \quad F=0 ; \quad G=f^{2}(u)\left(\dot{\varphi}^{2}(v)-\varphi^{2}(v)\right) .
$$

Since we are studying Lorentz surfaces, in the case $\dot{\varphi}^{2}(v)-\varphi^{2}(v)>0$ we assume that $f^{\prime}(u) g^{\prime}(u)>0$; in the case $\dot{\varphi}^{2}(v)-\varphi^{2}(v)<0$ we assume that $f^{\prime}(u) g^{\prime}(u)<0$.

We shall consider the tangent frame field defined by $X=\frac{z_{u}}{\sqrt{2 \varepsilon f^{\prime} g^{\prime}}}, Y=$ $\frac{z_{v}}{f \sqrt{\varepsilon\left(\dot{\varphi}^{2}-\varphi^{2}\right)}}$, where $\varepsilon=1$ in the case $\dot{\varphi}^{2}-\varphi^{2}>0, f^{\prime} g^{\prime}>0$, and $\varepsilon=-1$ in the case $\dot{\varphi}^{2}-\varphi^{2}<0, f^{\prime} g^{\prime}<0$. Thus we have $\langle X, X\rangle=-\varepsilon,\langle Y, Y\rangle=\varepsilon$, $\langle X, Y\rangle=0$. Let us choose the following normal frame field:

$$
\begin{aligned}
& n_{1}=\sqrt{\frac{\varepsilon f^{\prime}}{2 g^{\prime}}}\left(\varphi \cosh v e_{1}+\varphi \sinh v e_{3}+\frac{f^{\prime} \varphi^{2}-2 g^{\prime}}{2 f^{\prime}} \xi_{1}+\xi_{2}\right) ; \\
& n_{2}=\frac{1}{\sqrt{\varepsilon\left(\dot{\varphi}^{2}-\varphi^{2}\right)}}\left((\dot{\varphi} \sinh v+\varphi \cosh v) e_{1}+(\dot{\varphi} \cosh v+\varphi \sinh v) e_{3}+\varphi^{2} \xi_{1}\right),
\end{aligned}
$$

which satisfies $\left\langle n_{1}, n_{1}\right\rangle=\varepsilon,\left\langle n_{2}, n_{2}\right\rangle=-\varepsilon,\left\langle n_{1}, n_{2}\right\rangle=0$. Taking into account (3.3), we calculate the second partial derivatives of $z(u, v)$ :

$$
\begin{aligned}
z_{u u}= & f^{\prime \prime} \varphi \cosh v e_{1}+f^{\prime \prime} \varphi \sinh v e_{3}+\left(f^{\prime \prime} \frac{\varphi^{2}}{2}+g^{\prime \prime}\right) \xi_{1}+f^{\prime \prime} \xi_{2} ; \\
z_{u v}= & f^{\prime}(\dot{\varphi} \cosh v+\varphi \sinh v) e_{1}+f^{\prime}(\dot{\varphi} \sinh v+\varphi \cosh v) e_{3}+f^{\prime} \varphi \dot{\varphi} \xi_{1} ; \\
z_{v v}= & f((\ddot{\varphi}+\varphi) \cosh v+2 \dot{\varphi} \sinh v) e_{1}+f((\ddot{\varphi}+\varphi) \sinh v+2 \dot{\varphi} \cosh v) e_{3} \\
& +f\left(\dot{\varphi}^{2}+\varphi \ddot{\varphi}\right) \xi_{1} .
\end{aligned}
$$

The last equalities together with (3.4) imply

$$
\begin{array}{ll}
\left\langle z_{u u}, n_{1}\right\rangle=\frac{f^{\prime \prime} g^{\prime}-g^{\prime \prime} f^{\prime}}{\sqrt{2 \varepsilon f^{\prime} g^{\prime}}} ; & \left\langle z_{u u}, n_{2}\right\rangle=0 ; \\
\left\langle z_{u v}, n_{1}\right\rangle=0 ; & \left\langle z_{u v}, n_{2}\right\rangle=0 ; \\
\left\langle z_{v v}, n_{1}\right\rangle=-f \sqrt{\frac{\varepsilon f^{\prime}}{2 g^{\prime}}}\left(\dot{\varphi}^{2}-\varphi^{2}\right) ; & \left\langle z_{v v}, n_{2}\right\rangle=f \frac{\varphi \ddot{\varphi}-2 \dot{\varphi}^{2}+\varphi^{2}}{\sqrt{\varepsilon\left(\dot{\varphi}^{2}-\varphi^{2}\right)}} .
\end{array}
$$


Hence, we obtain

$$
\begin{aligned}
& h(X, X)=\varepsilon \frac{f^{\prime \prime} g^{\prime}-g^{\prime \prime} f^{\prime}}{\left(2 \varepsilon f^{\prime} g^{\prime}\right)^{\frac{3}{2}}} n_{1} ; \\
& h(X, Y)=0 ; \\
& h(Y, Y)=-\frac{1}{f} \sqrt{\frac{\varepsilon f^{\prime}}{2 g^{\prime}}} n_{1}-\varepsilon \frac{\varphi \ddot{\varphi}-2 \dot{\varphi}^{2}+\varphi^{2}}{f\left(\varepsilon\left(\dot{\varphi}^{2}-\varphi^{2}\right)\right)^{\frac{3}{2}}} n_{2} .
\end{aligned}
$$

Now, we shall consider the parametric lines of the meridian surface $\mathcal{M}_{m}$. The parametric $u$-line $v=v_{0}=$ const is given by

$$
c_{u}: z(u)=c \alpha f(u) e_{1}+c \beta f(u) e_{3}+\left(\frac{c^{2}}{2} f(u)+g(u)\right) \xi_{1}+f(u) \xi_{2},
$$

where $\alpha=\cosh v_{0}, \beta=\sinh v_{0}, c=\varphi\left(v_{0}\right)$. So, the unit tangent vector field $t_{c_{u}}$ of $c_{u}$ is:

$$
t_{c_{u}}=\frac{1}{\sqrt{2 \varepsilon f^{\prime} g^{\prime}}}\left(c \alpha f^{\prime} e_{1}+c \beta f^{\prime} e_{3}+\left(\frac{c^{2}}{2} f^{\prime}+g^{\prime}\right) \xi_{1}+f^{\prime} \xi_{2}\right) .
$$

We denote by $s$ the arc-length of $c_{u}$ and calculate the derivative

$$
\frac{d t_{c_{u}}}{d s}=\frac{t_{c_{u}}^{\prime}}{s^{\prime}}=\frac{\varepsilon\left(f^{\prime \prime} g^{\prime}-g^{\prime \prime} f^{\prime}\right)}{\left(2 \varepsilon f^{\prime} g^{\prime}\right)^{2}}\left(c \alpha f^{\prime} e_{1}+c \beta f^{\prime} e_{3}+\left(\frac{c^{2}}{2} f^{\prime}-g^{\prime}\right) \xi_{1}+f^{\prime} \xi_{2}\right) .
$$

Thus we obtain that the curvature of $c_{u}$ is $\frac{\varepsilon\left(f^{\prime \prime} g^{\prime}-g^{\prime \prime} f^{\prime}\right)}{\left(2 \varepsilon f^{\prime} g^{\prime}\right)^{\frac{3}{2}}}$. Finally, for each $v=$ const the parametric lines $c_{u}$ are congruent in $\mathbb{E}_{2}^{4}$. These curves are the meridians of $\mathcal{M}_{m}$. We denote $\kappa_{m}(u)=\frac{\varepsilon\left(f^{\prime \prime} g^{\prime}-g^{\prime \prime} f^{\prime}\right)}{\left(2 \varepsilon f^{\prime} g^{\prime}\right)^{\frac{3}{2}}}$.

Now, we shall consider the parametric $v$-lines of $\mathcal{M}_{m}$. Let $u=u_{0}=$ const and denote $a=f\left(u_{0}\right), b=g\left(u_{0}\right)$. The corresponding parametric $v$-line is given by

$$
c_{v}: z(v)=a \varphi(v) \cosh v e_{1}+a \varphi(v) \sinh v e_{3}+\left(a \frac{\varphi^{2}(v)}{2}+b\right) \xi_{1}+a \xi_{2} .
$$

The unit tangent vector field $t_{c_{v}}$ of $c_{v}$ is

$t_{c_{v}}=\frac{1}{\sqrt{\varepsilon\left(\dot{\varphi}^{2}-\varphi^{2}\right)}}\left((\dot{\varphi} \cosh v+\varphi \sinh v) e_{1}+(\dot{\varphi} \sinh v+\varphi \cosh v) e_{3}+\varphi \dot{\varphi} \xi_{1}\right)$.

Knowing the tangent vector field $t_{c_{v}}$ we calculate the curvature $\varkappa_{c_{v}}$ of $c_{v}$ and obtain that $\varkappa_{c_{v}}=\frac{\varphi \ddot{\varphi}-2 \dot{\varphi}^{2}+\varphi^{2}}{a\left(\varepsilon\left(\dot{\varphi}^{2}-\varphi^{2}\right)\right)^{\frac{3}{2}}}$. We denote $\kappa(v)=\frac{\varphi \ddot{\varphi}-2 \dot{\varphi}^{2}+\varphi^{2}}{\left(\varepsilon\left(\dot{\varphi}^{2}-\varphi^{2}\right)\right)^{\frac{3}{2}}}$. Then, for each $u=u_{0}=$ const the curvature of the corresponding parametric $v$-line is 
expressed as $\varkappa_{c_{v}}=\frac{1}{a} \kappa(v)$, where $a=f\left(u_{0}\right)$. Actually, $\kappa(v)$ is the curvature of the curve

$$
c: l=l(v)=\varphi(v) \cosh v e_{1}+\varphi(v) \sinh v e_{3}+\frac{\varphi^{2}(v)}{2} \xi_{1}+\xi_{2} .
$$

Consequently, formulas (3.5) take the form

$$
\begin{aligned}
& h(X, X)=\kappa_{m} n_{1} \\
& h(X, Y)=0 ; \\
& h(Y, Y)=-\frac{1}{f} \sqrt{\frac{\varepsilon f^{\prime}}{2 g^{\prime}}} n_{1}-\varepsilon \frac{\kappa}{f} n_{2} .
\end{aligned}
$$

It follows from (3.6) that the Gauss curvature $K$ of the meridian surface $\mathcal{M}_{m}$ is expressed as

$$
K=\varepsilon \frac{\kappa_{m}}{f} \sqrt{\frac{\varepsilon f^{\prime}}{2 g^{\prime}}}
$$

and the mean curvature vector field $H$ is given by

$$
H=-\frac{\varepsilon}{2}\left(\kappa_{m}+\frac{1}{f} \sqrt{\frac{\varepsilon f^{\prime}}{2 g^{\prime}}}\right) n_{1}-\frac{\kappa}{2 f} n_{2} .
$$

Without loss of generality we can assume that $2 \varepsilon f^{\prime} g^{\prime}=1$, which implies $\kappa_{m}=\frac{f^{\prime \prime}}{f^{\prime}}$. Hence,

$$
\begin{gathered}
K=\varepsilon \frac{f^{\prime \prime}}{f}, \\
H=-\frac{\varepsilon\left(f f^{\prime \prime}+\left(f^{\prime}\right)^{2}\right)}{2 f f^{\prime}} n_{1}-\frac{\kappa}{2 f} n_{2} .
\end{gathered}
$$

Now, using (3.4) and (3.6) we obtain that

$$
\begin{array}{rlrl}
\bar{\nabla}_{X} n_{1} & =\kappa_{m} X ; & \bar{\nabla}_{X} n_{2}=0 ; \\
\bar{\nabla}_{Y} n_{1}=\frac{1}{f} \sqrt{\frac{\varepsilon f^{\prime}}{2 g^{\prime}}} Y ; & \bar{\nabla}_{Y} n_{2}=-\varepsilon \frac{\kappa}{f} Y .
\end{array}
$$

Hence,

$$
\begin{array}{ll}
D_{X} n_{1}=0 ; & D_{X} n_{2}=0 ; \\
D_{Y} n_{1}=0 ; & D_{Y} n_{2}=0,
\end{array}
$$

where $D$ is the normal connection of the surface. The last equalities imply that the curvature of the normal connection of $\mathcal{M}_{m}$ is zero. So, we obtain the following statement. 
Proposition 3.1. The meridian surface $\mathcal{M}_{m}$, defined by (3.2), is a surface with flat normal connection.

In the next sections we will give the classification of the meridian surfaces with constant Gauss curvature, with parallel mean curvature vector field and with parallel normalized mean curvature vector field.

\section{MERIDIAN SURFACES With CONSTANT GAUss CURVATURE}

The study of surfaces with constant Gauss curvature is one of the essential topics in differential geometry. Surfaces with constant Gauss curvature in Minkowski space have drawn the interest of many geometers, see for example [14], [21], and the references therein.

Let $\mathcal{M}_{m}$ be a meridian surface, defined by (3.2). Then the Gauss curvature of $\mathcal{M}_{m}$ depends only on the meridian curve $m$ and is expressed by formula (3.7). First, we shall describe the meridian surfaces with zero Gauss curvature.

Theorem 4.1. Let $\mathcal{M}_{m}$ be a meridian surface, defined by (3.2). Then $\mathcal{M}_{m}$ is flat if and only if the meridian curve $m$ is given by

$$
f(u)=a u+b ; \quad g(u)=\frac{\varepsilon}{2 a} u+c,
$$

where $a=$ const $\neq 0, b=$ const, $c=$ const. In this case $\mathcal{M}_{m}$ is a developable ruled surface.

Proof. It follows from (3.7) that $K=0$ if and only if $f(u)=a u+b, a=$ const $\neq 0, b=$ const. Using that $2 \varepsilon f^{\prime} g^{\prime}=1$, we obtain $g(u)=\frac{\varepsilon}{2 a} u+c$, $c=$ const. Since in this case $\kappa_{m}=0$, then the meridian curve $m$ is part of a straight line, i.e. $\mathcal{M}_{m}$ lies on a ruled surface. Moreover, it follows from (3.9) that $\bar{\nabla}_{X} n_{1}=0 ; \bar{\nabla}_{X} n_{2}=0$, which implies that the normal space is constant at the points of a fixed straight line, and hence the tangent space is one and the same at the points of a fixed line. Consequently, $\mathcal{M}_{m}$ is part of a developable ruled surface.

The following theorem describes the meridian surfaces with constant nonzero Gauss curvature.

Theorem 4.2. Let $\mathcal{M}_{m}$ be a meridian surface, defined by (3.2). Then $\mathcal{M}_{m}$ has constant non-zero Gauss curvature $K$ if and only if the meridian curve $m$ is given by

$$
\begin{array}{llr}
f(u)=\alpha \cosh \sqrt{\varepsilon K} u+\beta \sinh \sqrt{\varepsilon K} u, & \text { if } \quad \varepsilon K>0 ; \\
f(u)=\alpha \cos \sqrt{-\varepsilon K} u+\beta \sin \sqrt{-\varepsilon K} u, & \text { if } & \varepsilon K<0,
\end{array}
$$


where $\alpha$ and $\beta$ are constants, $g(u)$ is defined by $g^{\prime}(u)=\frac{\varepsilon}{2 f^{\prime}(u)}$.

Proof. Using that the Gauss curvature is expressed by (3.7), we obtain that $K=$ const $\neq 0$ if and only if the function $f(u)$ satisfies the following differential equation

$$
f^{\prime \prime}(u)-\varepsilon K f(u)=0 .
$$

The general solution of this equation is given by (4.1), where $\alpha$ and $\beta$ are constants. Since we assume that $2 \varepsilon f^{\prime} g^{\prime}=1$, then the function $g(u)$ is determined by $g^{\prime}(u)=\frac{\varepsilon}{2 f^{\prime}(u)}$.

\section{5}

\section{MERIDIAN SURFACES WITH PARALLEL MEAN CURVATURE VECTOR FIELD}

Another basic class of surfaces in Riemannian and pseudo-Riemannian geometry are surfaces with parallel mean curvature vector field, since they are critical points of some functionals and play important role in differential geometry, the theory of harmonic maps, as well as in physics. The classification of surfaces with parallel mean curvature vector field in Riemannian space forms was given by Chen [5] and Yau [23]. Recently, spacelike surfaces with parallel mean curvature vector field in pseudo-Euclidean spaces with arbitrary codimension were classified in [8] and [9]. The classification of quasi-minimal surfaces with parallel mean curvature vector in $\mathbb{E}_{2}^{4}$ is given in [12]. Lorentz surfaces with parallel mean curvature vector field in arbitrary pseudo-Euclidean space $\mathbb{E}_{s}^{m}$ are studied in [10] and [13]. A nice survey on classical and recent results on submanifolds with parallel mean curvature vector in Riemannian manifolds as well as in pseudo-Riemannian manifolds is presented in [11].

In this section we shall describe the meridian surfaces with non-zero parallel mean curvature vector field, i.e. $H \neq 0$ and $D H=0$.

Under the assumption $2 \varepsilon f^{\prime} g^{\prime}=1$ the mean curvature vector field $H$ of the meridian surface $\mathcal{M}_{m}$ is given by formula (3.8). Using that $D_{X} n_{1}=D_{Y} n_{1}=$ $D_{X} n_{2}=D_{Y} n_{2}=0$, and $X=z_{u}, Y=\frac{z_{v}}{f \sqrt{\varepsilon\left(\dot{\varphi}^{2}-\varphi^{2}\right)}}$, we get

$$
\begin{aligned}
& D_{X} H=-\frac{\varepsilon}{2}\left(\frac{f f^{\prime \prime}+\left(f^{\prime}\right)^{2}}{f f^{\prime}}\right)^{\prime} n_{1}+\frac{\kappa f^{\prime}}{2 f^{2}} n_{2} \\
& D_{Y} H=-\frac{\kappa^{\prime}}{2 f^{2} \sqrt{\varepsilon\left(\dot{\varphi}^{2}-\varphi^{2}\right)}} n_{2} .
\end{aligned}
$$

Theorem 5.1. Let $\mathcal{M}_{m}$ be a meridian surface, defined by (3.2). Then $\mathcal{M}_{m}$ has parallel mean curvature vector field if and only if the curvature of $c$ is $\kappa=0$ 
and the meridian curve $m$ is determined by $f^{\prime}=\phi(f)$ where

$$
\phi(t)=\frac{a t^{2}+b}{2 t}, \quad a=\text { const } \neq 0, \quad b=\text { const },
$$

$g(u)$ is defined by $g^{\prime}(u)=\frac{\varepsilon}{2 f^{\prime}(u)}$. In this case $\mathcal{M}_{m}$ is a non-flat CMC surface lying in a hyperplane of $\mathbb{E}_{2}^{4}$.

Proof. Using formulas (5.1) we get that $\mathcal{M}_{m}$ has parallel mean curvature vector field if and only if the following conditions hold

$$
\begin{aligned}
& \left(\frac{f f^{\prime \prime}+\left(f^{\prime}\right)^{2}}{f f^{\prime}}\right)^{\prime}=0 ; \\
& \kappa f^{\prime}=0 ; \\
& \kappa^{\prime}=0 .
\end{aligned}
$$

Since $f^{\prime} \neq 0$, the equalities (5.2) imply that $\kappa=0$ and $\frac{f f^{\prime \prime}+\left(f^{\prime}\right)^{2}}{f f^{\prime}}=a=$ const. If $a=0$, then $H=0$, i.e. $\mathcal{M}_{m}$ is minimal. Since we consider non-minimal surfaces, we assume that $a \neq 0$. In this case the meridian curve $m$ is determined by the following differential equation:

$$
f f^{\prime \prime}+\left(f^{\prime}\right)^{2}=a f f^{\prime}, \quad a=\text { const } \neq 0 .
$$

The solutions of the last differential equation can be found as follows. Setting $f^{\prime}=\phi(f)$ in equation (5.3), we obtain that the function $\phi=\phi(t)$ is a solution of the equation

$$
\phi^{\prime}+\frac{1}{t} \phi=a
$$

The general solution of equation (5.4) is given by

$$
\phi(t)=\frac{a t^{2}+b}{2 t}, \quad b=\text { const. }
$$

In this case, the mean curvature vector field $H$ is given by $H=-\frac{\varepsilon a}{2} n_{1}$, and thus $\langle H, H\rangle=\frac{\varepsilon a^{2}}{4}=$ const. Hence, the surface $\mathcal{M}_{m}$ is a CMC surface. Moreover, since $\kappa=0$, from (3.9) it follows that $\bar{\nabla}_{X} n_{2}=0, \bar{\nabla}_{Y} n_{2}=0$. Hence, $\mathcal{M}_{m}$ lies in a 3 -dimensional constant hyperplane parallel to $\operatorname{span}\left\{X, Y, n_{1}\right\}$. The Gauss curvature $K \neq 0$, so $\mathcal{M}_{m}$ is a non-flat CMC surface lying in a hyperplane of $\mathbb{E}_{2}^{4}$.

Conversely, if the meridian curve $m$ is determined by (5.5), then by direct computation we get that $D_{X} H=D_{Y} H=0$, i.e. the surface has parallel mean curvature vector field. 
Theorem 5.1 shows that each meridian surface with parallel mean curvature vector field is a CMC surface and lies in a hyperplane of $\mathbb{E}_{2}^{4}$. So, we have the following result.

Corollary 5.2. There are no Lorentz meridian surfaces with parallel mean curvature vector field other than CMC surfaces lying in a hyperplane of $\mathbb{E}_{2}^{4}$.

Remark. The same result holds true for meridian surfaces lying on rotational hypersurfaces with spacelike or timelike axis [4].

\section{6 \\ MERIDIAN SURFACES WITH PARALLEL NORMALIZED MEAN CURVATURE VECTOR FIELD}

The class of surfaces with parallel mean curvature vector field is naturally extended to the class of surfaces with parallel normalized mean curvature vector field. A submanifold in a Riemannian manifold is said to have parallel normalized mean curvature vector field if the mean curvature vector is non-zero and the unit vector in the direction of the mean curvature vector is parallel in the normal bundle [6]. It is well known that submanifolds with non-zero parallel mean curvature vector field have parallel normalized mean curvature vector field. But the condition to have parallel normalized mean curvature vector field is much weaker than the condition to have parallel mean curvature vector field. For example, every surface in the Euclidean 3-space has parallel normalized mean curvature vector field but in the 4-dimensional Euclidean space, there exist abundant examples of surfaces which lie fully in $\mathbb{E}^{4}$ with parallel normalized mean curvature vector field, but not with parallel mean curvature vector field. In the pseudo-Euclidean space with neutral metric $\mathbb{E}_{2}^{4}$ the study of Lorentz surfaces with parallel normalized mean curvature vector field, but not parallel mean curvature vector field, is still an open problem.

In this section we give the classification of all meridian surfaces which have parallel normalized mean curvature vector field but not parallel $H$.

Let $\mathcal{M}_{m}$ be a meridian surface, defined by (3.2). The mean curvature vector field $H$ is given by formula (3.8). We assume that $\langle H, H\rangle \neq 0$, i.e. $\left(f f^{\prime \prime}+\right.$ $\left.\left(f^{\prime}\right)^{2}\right)^{2}-\kappa^{2} f^{\prime 2} \neq 0$.

If $\kappa=0$, then the normalized mean curvature vector field is $H_{0}=n_{1}$ and in view of (3.10) we have $D_{X} H_{0}=D_{Y} H_{0}=0$, i.e. $H_{0}$ is parallel in the normal bundle. We consider this case as trivial, since under the assumption $\kappa=0$ the surface $\mathcal{M}_{m}$ lies in a 3-dimensional hyperplane of $\mathbb{E}_{2}^{4}$ and every surface in 3 -dimensional space has parallel normalized mean curvature vector field. So, further we assume that $\kappa \neq 0$.

A unit normal vector field in the direction of $H$ is

$$
H_{0}=\frac{-1}{\sqrt{\left|\left(f f^{\prime \prime}+\left(f^{\prime}\right)^{2}\right)^{2}-\kappa^{2} f^{\prime 2}\right|}}\left(\left(f f^{\prime \prime}+\left(f^{\prime}\right)^{2}\right) n_{1}+\kappa f^{\prime} n_{2}\right) \text {. }
$$


For simplicity we denote

$$
A=\frac{-\left(f f^{\prime \prime}+\left(f^{\prime}\right)^{2}\right)}{\sqrt{\left|\left(f f^{\prime \prime}+\left(f^{\prime}\right)^{2}\right)^{2}-\kappa^{2} f^{\prime 2}\right|}}, \quad B=\frac{-\kappa f^{\prime}}{\sqrt{\left|\left(f f^{\prime \prime}+\left(f^{\prime}\right)^{2}\right)^{2}-\kappa^{2} f^{\prime 2}\right|}},
$$

so, the normalized mean curvature vector field is expressed as $H_{0}=A n_{1}+B n_{2}$. Then from equalities (6.1) and (3.10) we get

$$
\begin{aligned}
& D_{X} H_{0}=X(A) n_{1}+X(B) n_{2} ; \\
& D_{Y} H_{0}=Y(A) n_{1}+Y(B) n_{2} .
\end{aligned}
$$

Theorem 6.1. Let $\mathcal{M}_{m}$ be a meridian surface, defined by (3.2). Then $\mathcal{M}_{m}$ has parallel normalized mean curvature vector field but not parallel mean curvature vector if and only if one of the following cases holds:

(i) $\kappa \neq 0$ and the meridian curve $m$ is defined by

$$
f(u)=\sqrt{a u+b}, \quad g(u)=\frac{2}{3 a^{2}}(a u+b)^{\frac{3}{2}}+c,
$$

where $a=$ const $\neq 0, b=$ const, $c=$ const.

(ii) $\kappa=$ const $\neq 0$ and the meridian curve $m$ is determined by $f^{\prime}=$ $\phi(f)$ where

$$
\phi(t)=\frac{c t+b}{t}, \quad c=\text { const } \neq 0, c^{2} \neq \kappa^{2}, b=\text { const },
$$

$g(u)$ is defined by $g^{\prime}(u)=\frac{\varepsilon}{2 f^{\prime}(u)}$.

Proof. Let $\mathcal{M}_{m}$ be a surface with parallel normalized mean curvature vector field, i.e. $D_{X} H_{0}=0, D_{Y} H_{0}=0$. Then from (6.2) it follows that $A=$ const, $B=$ const. Hence,

$$
\begin{aligned}
& \frac{-\left(f f^{\prime \prime}+\left(f^{\prime}\right)^{2}\right)}{\sqrt{\left|\left(f f^{\prime \prime}+\left(f^{\prime}\right)^{2}\right)^{2}-\kappa^{2} f^{\prime 2}\right|}}=\alpha=\text { const } \\
& \frac{-\kappa f^{\prime}}{\sqrt{\left|\left(f f^{\prime \prime}+\left(f^{\prime}\right)^{2}\right)^{2}-\kappa^{2} f^{\prime 2}\right|}}=\beta=\text { const } .
\end{aligned}
$$

We have the following two cases.

Case (i): $f f^{\prime \prime}+\left(f^{\prime}\right)^{2}=0$. In this case, from (3.8) we get that the mean curvature vector field is $H=-\frac{\kappa}{2 f} n_{2}$ and the normalized mean curvature vector field is $H_{0}=n_{2}$. Since we study surfaces with $\langle H, H\rangle \neq 0$, we get $\kappa \neq 0$. The solution of the differential equation $f f^{\prime \prime}+\left(f^{\prime}\right)^{2}=0$ is given by the formula $f(u)=\sqrt{a u+b}$, where $a=$ const $\neq 0, b=$ const. Using that $g^{\prime}(u)=\frac{\varepsilon}{2 f^{\prime}(u)}$, we obtain $g(u)=\frac{2}{3 a^{2}}(a u+b)^{\frac{3}{2}}+c$, where $c=$ const. 
Case (ii): $f f^{\prime \prime}+\left(f^{\prime}\right)^{2} \neq 0$ in an interval $\tilde{I} \subset I \subset \mathbb{R}$. Then, from (6.3) we get

$$
\frac{\alpha}{\beta} \kappa=\frac{f f^{\prime \prime}+\left(f^{\prime}\right)^{2}}{f^{\prime}}, \quad \alpha \neq 0, \beta \neq 0 .
$$

Since the left-hand side of equality (6.4) is a function of $v$, the right-hand side of (6.4) is a function of $u$, we obtain that

$$
\begin{aligned}
& \frac{f f^{\prime \prime}+\left(f^{\prime}\right)^{2}}{f^{\prime}}=c, \quad c=\text { const } \neq 0 ; \\
& \kappa=\frac{\beta}{\alpha} c .
\end{aligned}
$$

In this case we have $\langle H, H\rangle=\frac{\varepsilon\left(c^{2}-\kappa^{2}\right)}{4 f^{2}}$. Since we study surfaces with $\langle H, H\rangle \neq 0$, we get $c^{2} \neq \kappa^{2}$. The meridian curve $m$ is determined by the following differential equation:

$$
f f^{\prime \prime}+\left(f^{\prime}\right)^{2}=c f^{\prime}
$$

Setting $f^{\prime}=\phi(f)$ in equation (6.5), we obtain that the function $\phi=\phi(t)$ satisfies

$$
\phi^{\prime}+\frac{1}{t} \phi=\frac{c}{t},
$$

whose general solution is $\phi(t)=\frac{c t+b}{t}, b=$ const.

Conversely, if one of the cases (i) or (ii) stated in the theorem holds true, then by direct computation we get that $D_{X} H_{0}=D_{Y} H_{0}=0$, i.e. the surface has parallel normalized mean curvature vector field. Moreover, in case (i) we have

$$
D_{X} H=\frac{\kappa f^{\prime}}{2 f^{2}} n_{2} ; \quad D_{Y} H=-\frac{\kappa^{\prime}}{2 f^{2} \sqrt{\varepsilon\left(\dot{\varphi}^{2}-\varphi^{2}\right)}} n_{2},
$$

which implies that $H$ is not parallel in the normal bundle, since $\kappa \neq 0, f^{\prime} \neq 0$. In case (ii) we get

$$
D_{X} H=\frac{\varepsilon c f^{\prime}}{2 f^{2}} n_{1}+\frac{\kappa f^{\prime}}{2 f^{2}} n_{2} ; \quad D_{Y} H=0,
$$

and again we have that $H$ is not parallel in the normal bundle.

Remark. Theorem 6.1 gives examples of Lorentz surfaces in the pseudoEuclidean space $\mathbb{E}_{2}^{4}$ which have parallel normalized mean curvature vector field but not parallel mean curvature vector field.

\section{ACKNOWLEDGEMENTS}

The author is partially supported by the Bulgarian National Science Fund, Ministry of Education and Science of Bulgaria under contract DFNI-I 02/14. 


\section{REFERENCES}

[1] Arslan K., Bulca B., Milousheva V., Meridian surfaces in $\mathbb{E}^{4}$ with pointwise 1-type Gauss map, Bull. Korean Math. Soc., 51 (2014), 911-922.

[2] Arslan K., Milousheva V., Meridian surfaces of elliptic or hyperbolic type with pointwise 1-type Gauss map in Minkowski 4-space, Taiwanese J. Math., 20 (2016), 311-332.

[3] Bulca B., Milousheva V., Meridian surfaces with constant mean curvature in pseudo-Euclidean 4-space with neutral metric, Mediterr. J. Math., 14 (2017), 14: 48.

[4] Bulca B., Milousheva V., Meridian surfaces with parallel normalized mean curvature vector field in pseudo-Euclidean 4-space with neutral metric, Int. Jour. Geom. 6 (2017), 67-84

[5] Chen B.-Y., Geometry of Submanifolds, Marcel Dekker, Inc., New York 1973.

[6] Chen B.-Y., Surfaces with parallel normalized mean curvature vector, Monatsh. Math., 90 (1980), 185-194.

[7] Chen B.-Y., Classification of marginally trapped surfaces of constant curvature in Lorentzian complex plane, Hokkaido Math. J., 38 (2009), 361-408.

[8] Chen B.-Y., Classification of spatial surfaces with parallel mean curvature vector in pseudo-Euclidean spaces with arbitrary codimension, J. Math. Phys., 50 (2009), 043503.

[9] Chen B.-Y., Complete classification of spatial surfaces with parallel mean curvature vector in arbitrary non-flat pseudo-Riemannian space forms, Cent. Eur. J. Math., 7 (2009), 400-428.

[10] Chen B.-Y., Complete classification of Lorentz surfaces with parallel mean curvature vector in arbitrary pseudo-Euclidean space, Kyushu J. Math., 64 (2010), 261-279.

[11] Chen B.-Y., Submanifolds with parallel mean curvature vector in Riemannian and indefinite space forms, Arab J. Math. Sci., 16 (2010), 1-46.

[12] Chen B.-Y., Garay O., Classification of quasi-minimal surfaces with parallel mean curvature vector in pseudo-Euclidean 4-space $\mathbb{E}_{2}^{4}$, Result. Math., 55 (2009), 23-38.

[13] Fu Y., Hou Z.-H., Classification of Lorentzian surfaces with parallel mean curvature vector in pseudo-Euclidean spaces, J. Math. Anal. Appl., 371 (2010), 25-40. 
[14] Gálvez J., Martínez A., Milán F., Complete constant Gaussian curvature surfaces in the Minkowski space and harmonic diffeomorphisms onto the hyperbolic plane, Tohoku Math. J., 55 (2003), 467-476.

[15] Ganchev G., Milousheva V., Invariants and Bonnet-type theorem for surfaces in $\mathbb{R}^{4}$, Cent. Eur. J. Math., 8 (2010), 993-1008.

[16] Ganchev G., Milousheva V., An invariant theory of marginally trapped surfaces in the four-dimensional Minkowski space, J. Math. Phys., 53 (2012), Article ID: 033705, 15 pp.

[17] Ganchev G., Milousheva V., Special classes of meridian surfaces in the fourdimensional Euclidean space, Bull. Korean Math. Soc., 52 (2015), 20352045 .

[18] Ganchev G., Milousheva V., Meridian surfaces of elliptic or hyperbolic type in the four-dimensional Minkowski space, Math. Commun., 21 (2016), 1-21.

[19] Ganchev G., Milousheva V., Marginally trapped meridian surfaces of parabolic type in the four-dimensional Minkowski space, Int. J. Geom. Methods Mod. Phys., 10 (2013), Article ID: 1350060, 17 pp.

[20] Ganchev G., Milousheva V., Meridian surfaces of parabolic type in the fourdimensional Minkowski space, in Geometry, Integrability and Quantization, I. Mladenov, G. Meng and A. Yoshioka (Eds), Avangard Prima, 2016, 243255.

[21] López R., Surfaces of constant Gauss curvature in Lorentz-Minkowski threespace, Rocky Mountain J. Math., 33 (2003), 971-993.

[22] O'Neill M., Semi-Riemannian geometry with applications to relativity, Academic Press, London 1983.

[23] Yau, S., Submanifolds with constant mean curvature, Amer. J. Math., 96 (1974), 346-366. 